\section{The UK National Cyclodiode Laser Survey}

Keywords: glaucoma; raised intraocular pressure; laser coagulation; ciliary body

\section{Introduction}

Ciliary body ablation in the management of refractory glaucoma is a well-recognised therapeutic option to lower intraocular pressure (IOP). Traditionally, cyclodestructive procedures had been reserved for end-stage glaucoma resistant to conventional management. However, the original techniques of cyclodiathermy ${ }^{1}$ and cryotherapy ${ }^{2}$ have now been supplanted by laser photocoagulation, initially with the 1064-nm neodymium/YAG ${ }^{3}$ and more recently with the transscleral $810-\mathrm{nm}$ semiconductor diode laser. ${ }^{4}$ The latter affords selective absorption of its wavelength by the uveal melanin, deeper ciliary body coagulation, ${ }^{5}$ and a lower incidence of complications, such as phthisis, uveitis, visual loss, and pain. ${ }^{6}$ This has made the 810-nm semiconductor diode laser a more commonly used treatment modality in the management of glaucoma. Most commonly transscleral diode laser cyclophotocoagulation (cyclodiode) is performed with the Oculight SLx diode laser system and contact G-probe (Iris Medical Instruments, Mountain View, CA, USA). The G-probe is applied tangential to the corneoscleral limbus, and laser energy is focused at a point corresponding to the pars plicata of the ciliary body, $1-2 \mathrm{~mm}$ behind the limbus (Figure 1). Some surgeons may use a light source to transilluminate to identify the location of the ciliary body more accurately.

There is currently a lack of consensus on the optimum practice of cyclodiode laser treatment in the United Kingdom. This cross-sectional study sought to ascertain (1) current practice patterns of cyclodiode laser and (2) attitudes and perspectives towards its use amongst consultant ophthalmologists in the United Kingdom. 


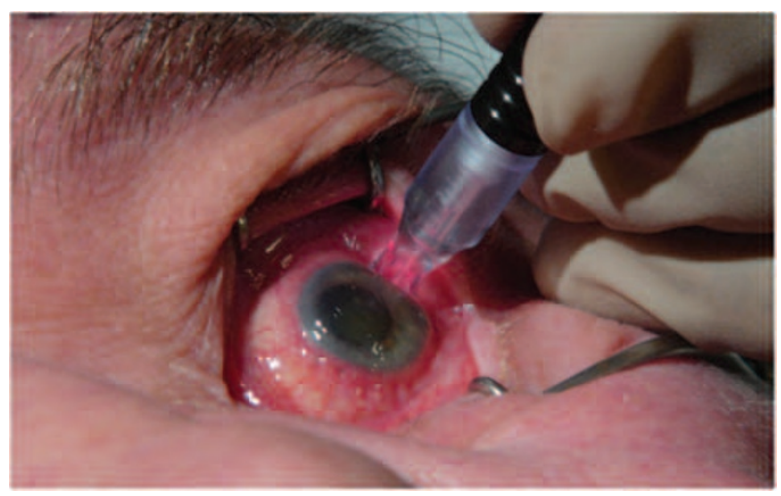

Figure 1 Diode laser cycloablation performed using a G-probe.

\section{Methods}

Questionnaires were distributed in both paper and online forms. Postal details of currently practising UK consultant ophthalmologists were obtained from the Royal College of Ophthalmologists. Email addresses were derived using the standard format for a UK National Health Service consultant of firstname.surname@trust.nhs.uk. For email addresses that bounced the consultant's secretary was contacted for an alternative email address. All data were treated anonymously and were returned using an individualised secure SSL internet server weblink embedded in the invitation email.

A 31-question survey was designed using a commercial website (http:/ /www.surveymonkey.com) and emailed to all the 952 UK consultants in October 2008. After 2 weeks, a reminder was sent to the non-responders. Six weeks following the online invitation, paper questionnaires were sent to the remaining 726 non-responders. Paper questionnaires were returned to the author in a prepaid self-addressed envelope and manually entered into the website database alongside the online results. All information gathered was treated in strict accordance with the Privacy Act 2001. Data was analysed using surveymonkey.com analysis tools. Statistical analyses were performed using SPSS software version 10.1 (SPSS Inc., Chicago, IL, USA). $P$-values of $<0.05$ were considered to indicate statistical significance. Where responders skipped questions, they were omitted from percentage calculations for that particular question.

The first seven questions centred on demographic data and asked whether the consultant personally performed cyclodiode laser. The next 17 questions ascertained current practice techniques and attitudes to various aspects of cyclodiode laser, including type of anaesthesia, transillumination, and power settings. Factors that may influence the power settings during cyclodiode laser

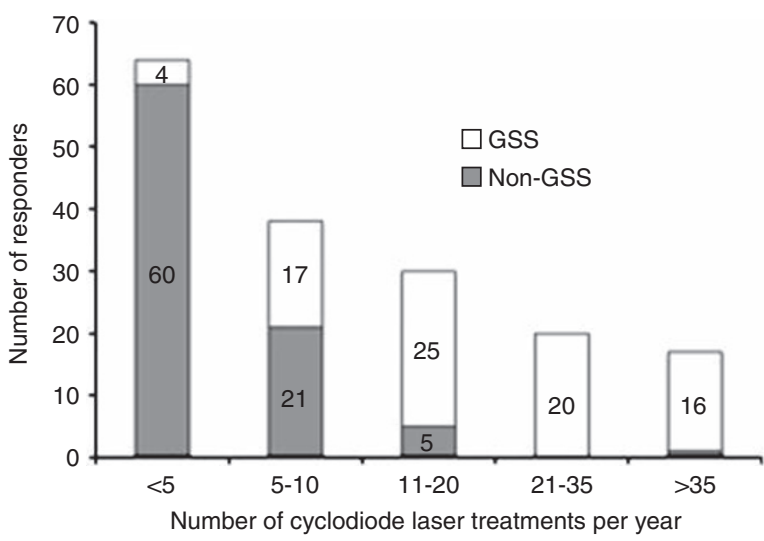

Figure 2 Number of cyclodiode laser treatments performed per year. GSS, glaucoma subspecialists $(n=82)$; Non-GSS, nonglaucoma subspecialists $(n=87)$.

treatment were also assessed. The final questions looked at post-operative management, including follow-up time periods, the use of steroids, and repeat laser treatment.

\section{Results}

A total of 510 participants (53.6\%) responded, of which 492 fully completed the survey. Responders from all deaneries of the United Kingdom were represented in the survey.

A total of 180 participants $(35.3 \%)$ reported performing cyclodiode laser treatment, of which 84 (46.7\%) considered themselves glaucoma subspecialists (GSS). The information from the group that performed cyclodiode laser treatment $(35.3 \%)$ was used in the subsequent analysis.

Cyclodiode laser is performed in all sizes of unit with no bias towards larger units. It is performed by both GSS and non-GSS; however, there was a strong trend for the GSS to perform more procedures than the non-GSS per year (Figure 2). The mean number of procedures per year for the GSS was 20.1 vs 4.8 for the non-GSS $(P<0.0001$, Mann-Whitney $U$-test).

\section{Technique}

All responders performed transscleral cyclodiode $(n=169)$ of which $7(4.1 \%)$ also performed endoscopic cyclophotocoagulation. In all, $97 \%$ of the responders performed all cyclodiode laser procedures in a theatre setting. The full range of anaesthetic options were variously employed with responders given more than one option as to their preferred choice. In all, 50.3\% preferred using subtenon anaesthetic, $46.1 \%$ peribulbar, $12.6 \%$ retrobulbar, $4.2 \%$ topical, $2.4 \%$ subconjunctival, and $31.1 \%$ general anaesthetic $(n=167)$. 
Transillumination of the globe is performed routinely by $64.9 \%(n=168)$. For the GSS, this was $78 \%(n=82)$ and in non-GSS $52.3 \%\left(n=86 ; P=0.0009, \chi^{2}\right.$ test). Initial median power settings used were $1500 \pm 331 \mathrm{~mW}$ (range $1000-3000 \mathrm{~mW}$ ) and $2000 \pm 466 \mathrm{~ms}$ (range 500-2500 ms). The average number of applications delivered per sitting was $23.1 \pm 9.8$ (mean \pm SD) applications (range 8-50) in a seeing eye $v s 27 \pm 10.2$ (range 10-60) in a blind eye $(P<0.0001$, Student's $t$-test). For the GSS, in a seeing eye, the average number of applications was $25.5 \pm 9.6$ and for the non-GSS 20.6 \pm 8.2 . In a seeing eye, therefore, the GSS perform, on average, 4.9 more applications per eye than the non-GSS ( $P=0.0013$, Mann-Whitney U-test)

When performing cyclodiode, $19.4 \%$ of the responders considered it necessary to hear a 'pop'. Only $12.3 \%$ of the GSS vs $26.2 \%$ of the non-GSS considered is necessary to hear a 'pop' $(P=0.03)$. The number of 'pops' considered safe during cyclodiode laser treatment varied amongst responders and ranged from 0 to 10 as illustrated in Figure 3. Nine responders (5.9\%) considered 10 'pops' during laser treatment to be safe. On hearing a 'pop', 75.2\% of the responders reduce the power $(n=161)$.

When applying cyclodiode laser, over $85 \%$ of the responders avoid the 3 o'clock and 9 o'clock meridia where the long ciliary nerves are located, potentially reducing post-operative pain. In all, $18 \%$ of the responders avoid the superotemporal area $(n=134)$, hence preserving the superior conjunctiva and sclera for possible future filtration surgery. There were no significant differences between the GSS and non-GSS.

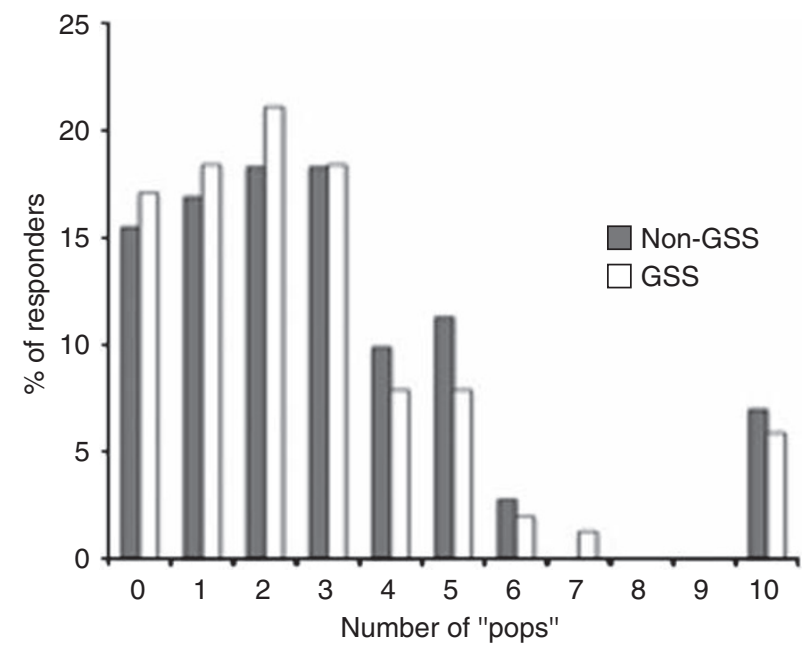

Figure 3 Number of 'pops' considered safe during cyclodiode laser treatment. GSS, glaucoma subspecialists $(n=81)$; Non-GSS, non-glaucoma subspecialists $(n=71)$.

\section{Factors influencing application of cyclodiode}

Responders were asked whether they varied their cyclodiode settings in different clinical situations. In the majority of cases, responders used their standard settings (Table 1). However, there was a particular tendency for the GSS to reduce their settings in patients with uveitic glaucoma $(43.1 \%)$ compared with the non-GSS $(16.9 \%, P=0.0014)$. In eyes with no visual potential, $29.7 \%$ of the GSS increased their settings $v$ s $11.8 \%$ of the non-GSS $(P=0.013)$. There was a tendency to lower settings in cases of previous cyclodiode, pigmented ethnicity and seeing eyes, and to increase settings in neovascular glaucoma but with no significant variation between the GSS and non-GSS.

When asked about bilateral cyclodiode laser treatment, $32.7 \%$ of the responders would consider performing this at one sitting $(n=159)$. For the GSS, $40.8 \%$ would perform bilateral cyclodiode $v$ s $25.3 \%$ of the non-GSS $(P=0.043)$. When asked about combined phacoemulsification and cyclodiode in one sitting, $22.3 \%$ of the responders said they would consider performing this with no statistically significant difference between the GSS and non-GSS.

Cyclodiode laser is performed in patients of all ages in the United Kingdom and at any level of visual acuity. In all, $49 \%$ of responders reported performing cyclodiode in any age group (81/165), whereas $60 \%$ $(98 / 162)$ performed cyclodiode at any visual acuity. Only a small percentage reserve cyclodiode for visual acuities of $6 / 60$ or worse $(12.3 \%, 20 / 162)$. A very small proportion of responders reserve cyclodiode for those of 66 years and over $(4.2 \%, 7 / 165)$. However, $28.1 \%$ $(39 / 139)$ of the responders felt that cyclodiode was most effective in the over 66 years of age group.

Table 1 Factors influencing cyclodiode settings (\%)

\begin{tabular}{|c|c|c|c|c|c|c|}
\hline & \multicolumn{2}{|c|}{ Lower } & \multicolumn{2}{|c|}{ Standard } & \multicolumn{2}{|c|}{ Higher } \\
\hline & GSS & $\begin{array}{c}\text { Non- } \\
\text { GSS }\end{array}$ & GSS & $\begin{array}{l}\text { Non- } \\
\text { GSS }\end{array}$ & GSS & $\begin{array}{l}\text { Non- } \\
\text { GSS }\end{array}$ \\
\hline Previous cyclodiode & 18.1 & 18.8 & 76.4 & 75.4 & 5.6 & 5.8 \\
\hline Pigmented ethnicity & 27.0 & 36.6 & 71.6 & 59.2 & 1.4 & 4.2 \\
\hline Increasing IOP & 1.3 & 0 & 88.0 & 89.9 & 10.7 & 10.1 \\
\hline Uveitic glaucoma & $43.1^{*}$ & 16.9 & 56.9 & 88.0 & 0.0 & 3.1 \\
\hline Rubeotic glaucoma & 5.4 & 1.4 & 74.3 & 81.4 & 20.3 & 17.1 \\
\hline Good visual potential & 24.0 & 17.1 & 74.7 & 82.9 & 1.3 & 0.0 \\
\hline No visual potential & 0 & 0 & 70.3 & 88.2 & $29.7^{* *}$ & 11.8 \\
\hline
\end{tabular}

Abbreviations: GSS, glaucoma subspecialists $(n=75)$; IOP, intraocular pressure; Non-GSS, non-glaucoma subspecialists $(n=72)$.

Respondents were asked whether they would use standard, lower, or higher settings.

${ }^{*} P=0.0014,{ }^{* *} P=0.013$, Fisher's exact test (comparing GSS with non-GSS). 


\section{Post-operative management}

Post-operatively, $46.6 \%$ give periocular steroids, of which $81.3 \%$ give subconjunctival betamethasone sodium phosphate $0.1 \%(n=161)$. Topical steroids are routinely prescribed by $98.8 \%$ of the practitioners with the modal steroids being prednisolone $1 \%$ or dexamethasone $0.1 \%$, four times daily for $2-4$ weeks.

The time until the first post-operative IOP check varied from $1 \mathrm{~h}$ to 6 weeks (Figure 4 ). The majority of responders checked the IOP at 1-2 weeks (62.2\%). In all, $36 \%$ of the non-GSS checked the IOP within 3 days of treatment compared with $17 \%$ of the GSS. Should the IOP not settle, most repeat treatments are done at 6 weeks $(47.4 \%)$ or 3 months $(40.3 \%, n=154)$.

Figure 5 illustrates the number of repeat cyclodiode treatments considered acceptable for the GSS and non-GSS. The mean number considered acceptable by the GSS was $3.67 \pm 1.90$ and $2.67 \pm 1.2$ for the non-GSS.

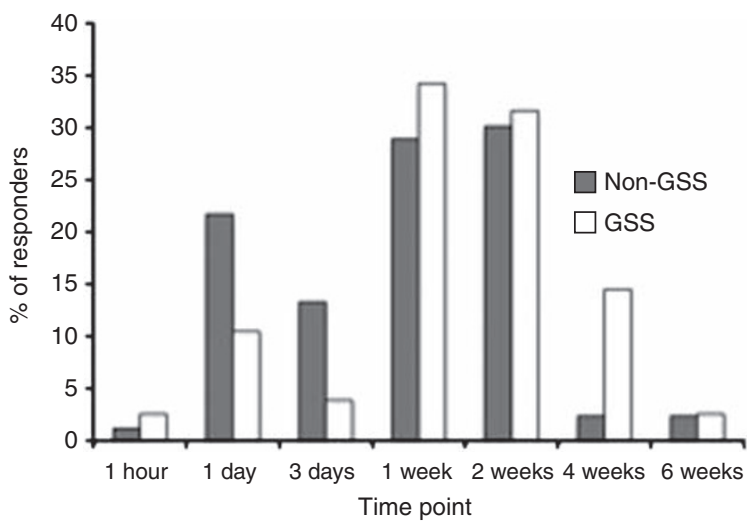

Figure 4 Time point when first post-operative IOP check is performed. GSS, glaucoma subspecialists $(n=76)$; Non-GSS, non-glaucoma subspecialists $(n=83)$.

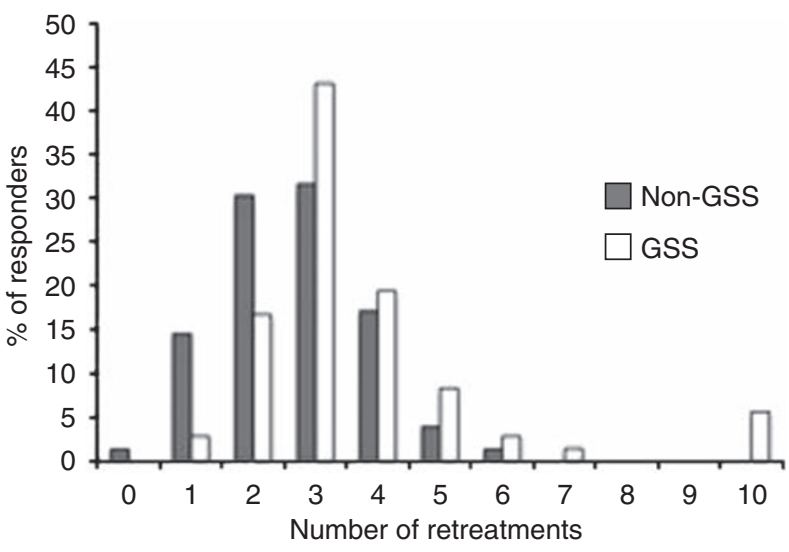

Figure 5 Number of repeat cyclodiode laser treatments considered acceptable. GSS, glaucoma subspecialists $(n=72)$; Non-GSS, non-glaucoma subspecialists $(n=76)$.
This difference was statistically significant $(P=0.0003)$ and remained so when the four outliers in the GSS group (10 retreatments) were excluded from the analysis $(P=0.002)$.

\section{Discussion}

There is a wide variation in the practice of cyclodiode laser amongst UK consultant ophthalmologists. We have identified some statistically significant differences between the GSS and non-GSS. The GSS are more likely to transilluminate the globe, apply more applications in the seeing eye, lower power settings when treating uveitic glaucoma, and increase power settings in eyes with no visual potential while performing cyclodiode laser treatment.

There is a relative lack of data regarding the optimal treatment settings and number of applications per session for cyclodiode laser. It is difficult and often misleading to attempt valid comparisons between different studies, because of a lack of standardisation in study designs and follow-up periods, referral patterns, and patients' demographics. The traditional values of $1500 \mathrm{~mW}$ and $1500 \mathrm{~ms}$ were taken from histological studies performed on a limited number of cadaveric eyes and assessing morphological changes. ${ }^{5,7,8}$ The present study provides the first consensus information on the clinical use of cyclodiode laser with 165 specialists reporting their standard settings.

Taking the responders' initial power settings, the mean total energy delivered per session in this survey was $71.7 \pm 30.9 \mathrm{~J}$ in a seeing eye and $84.4 \pm 34.2 \mathrm{~J}$ in a blind eye. A clear linear relationship between total energy delivered and percentage IOP reduction is yet to be demonstrated in published case series. However, there does seem to be a correlation between higher power settings and a higher incidence of hypotony. In a retrospective analysis of 263 eyes undergoing transscleral cyclodiode, Murphy et $a l^{9}$ report a $52.6 \%$ fall in post-treatment IOP following a mean energy delivery of $104.1 \pm 37.5 \mathrm{~J}$. The incidence of hypotony and phthisis was 9.5 and $5.3 \%$, respectively. Conversely, Spencer and Vernon ${ }^{10}$ report a $50.3 \%$ fall in IOP with $3.4 \%$ incidence of hypotony following a standardised protocol of only $56 \mathrm{~J}$ per treatment session. However, in this series, $45 \%$ of the patients required retreatment. Bloom et al ${ }^{11}$ also reported a low incidence of hypotony (1\%) in their retrospective analysis of 209 eyes in which the mean energy delivery was $90 \mathrm{~J}$. In their series, $49 \%$ of the eyes required retreatment with some loss of vision occurring in $50 \%$ of those receiving three or more treatments. It is difficult to ascertain, however, whether this was secondary to initial ocular pathology causing the secondary glaucoma, progression of glaucomatous visual field loss from poor IOP control, 
or secondary to complications of cyclodiode. In the current survey, $7 \%$ of the responders, all GSS, would consider performing 10 or more repeat treatments.

Ciliary body transillumination has been proposed as a useful technique to ensure cyclodiode laser treatment is directed towards the pars plicata. It has been advocated in the context of congenital glaucoma, high myopia, and abnormal limbal anatomy. Previous light microscopy studies have suggested optimal probe placement in contact cycloablation to be $0.5-1.0 \mathrm{~mm}$ posterior to the corneoscleral limbus. ${ }^{12,13}$ However, ciliary body position has been shown to vary with axial length, between each quadrant of the eye, and between individual glaucoma patients. ${ }^{14}$ Furthermore, endoscopic flourescein angiography of the ciliary body in patients with failed 360-degree transscleral diode cycloablation has demonstrated that the vast majority of patients actually experienced less than 90 degrees of cyclodestruction. ${ }^{15}$ It could therefore be argued that ciliary body transillumination should be performed continuously during every shot of cyclodiode laser. However, only two-thirds of responders report routinely performing transillumination with almost $80 \%$ of the GSS and just over half of all the non-GSS undertaking routine ciliary body transillumination $(P=0.0006)$.

Audible 'pops' during cyclodiode laser treatment are thought to represent intraocular uveal microexplosions when the threshold between coagulation and explosive tissue disintegration is exceeded. ${ }^{16}$ The presence of a 'pop' may indicate optimal probe placement and almost $20 \%$ of the responders consider it necessary to hear a 'pop'. The effect of 'pops' is reported to be undesirable, however, and if excessive, can be associated with post-operative iridocyclitis, hyphaema, pain, cystoid macular oedema, visual loss, hypotony, and pthisis. ${ }^{11}$ Despite this, almost $6 \%$ of the responders considered 10 'pops' during cycloablation to be safe. Nevertheless, the majority of the responders $(75.2 \%)$ would reduce laser power on hearing 'pops'.

Limited knowledge exists on how to adjust power settings to various glaucoma aetiologies that would help ensure optimum treatment. This study has revealed a tendency to reduce settings in uveitic glaucoma, pigmented ethnicity, previous cyclodiode laser treatment, and eyes with visual potential. Murphy et $a l^{9}$ reported a higher risk of hypotony in uveitic glaucoma ( 3 of the 16 eyes, 18.8\%) and advocated a more cautious approach in these patients. The UK practice seems to reflect this advice, significantly more so amongst the GSS than the non-GSS $(P=0.0014)$. Previous studies have also reported lower retreatment rates in uveitic glaucoma, suggesting uveitic eyes are particularly sensitive to cyclodiode. ${ }^{17,18}$ The longstanding cyclitis in uveitic eyes may result in compromised ciliary body function, as a result of which the remaining functional ciliary body responds readily to cycloablation.

The uptake of laser energy is thought to be related to the amount of ocular pigmentation, which may explain the tendency for the GSS and non-GSS to reduce power settings in pigmented ethnicity. ${ }^{19}$ However, a clear relationship between pigmentation and IOP response in cyclodiode remains to be demonstrated. Endoscopy of the ciliary body has identified no visible difference between the pigmentation of the ciliary body in blue-green eyes $v$ s brown eyes. ${ }^{20}$ However, Gasterland and Pollock, ${ }^{21}$ and Kosoko et al ${ }^{22}$ reported that patients with dark brown iris colour were more likely to have audible 'pops' during cyclodiode than patients with less pigmented iris colours, suggesting better energy absorption in darker irides. Conversely, other studies have found no difference in the number of 'pops' between different iris colours. ${ }^{11,16}$

This study has shown a tendency to increase power in neovascular glaucoma and in patients with increasing pre-treatment IOPs. This was consistent across both the GSS and non-GSS. However, previous studies have found a higher risk of hypotony in eyes with neovascular glaucoma, especially with higher pre-treatment IOP and those receiving higher energy treatments. ${ }^{9}$ High pre-treatment IOP may cause ciliary body ischaemia, which in turn may lead to ciliary body shutdown following cyclodiode laser. It has therefore been proposed a more cautious approach in neovascular glaucoma with lower total energy delivery per session and more treatment sessions based on the IOP response especially in those patients with good visual potential.

In this survey, only one-fifth of the responders would consider performing phacoemulsification and cyclodiode in one sitting. Recent cataract surgery is a well-known risk factor for trabeculectomy failure. ${ }^{23}$ Cataract surgery causes prolonged breakdown of the blood aqueous barrier compared with eyes with trabeculectomy alone. ${ }^{24}$ This persistent breakdown with serum-like components in the aqueous may upregulate fibrogenic cytokines production and has been shown to reduce mitomycin $c$ cytotoxicity to fibroblasts. ${ }^{25}$ In such cases, combined phacoemulsification with cyclodiode may be a more attractive option in temporarily controlling the IOP while delaying filtration surgery until anterior chamber inflammation has settled.

Cyclodiode laser is commonly used for refractory glaucoma with poor visual potential; however, it is becoming an increasingly attractive alternative in the management of less advanced glaucoma with good visual potential. Although there is consensus in some areas, this survey exposes wide variations in practice and perceptions of diode laser cycloablation amongst the GSS and non-GSS in the United Kingdom. The most 
frequently used practice may not necessarily be optimal practice. It may be of some value to consider individualising parameters for each patient based on various factors, including type of glaucoma, severity of visual loss, height of IOP, patient age, and number of previous cyclophotocoagulation treatments. Further guidance is required as to optimum energy settings and factors predicting response. The results of this survey would be helpful in developing local and national guidelines on the use of cyclodiode laser treatment.

\section{Summary}

\section{What was known before}

- Diode laser cycloablation is an effective technique in lowering intraocular pressure in refractory glaucoma.

- There are no national guidelines on the uniform practice of the technique.

\section{What this study adds}

- Although there is agreement in some areas of cyclodiode practice in the United Kingdom, a wide variation in practice exists.

- Significant differences in practice are present between glaucoma specialists and non-glaucoma specialists.

- Glaucoma specialists are more likely to transilluminate the globe, apply more shots in the blind eye, and lower power setting in uveitic glaucoma.

- This study will help streamline cyclodiode practice nationally and will encourage the development of guidelines.

\section{Conflict of interest}

The authors declare no conflict of interest.

\section{References}

1 Vogt A. Cyclodiathermypuncture in cases of glaucoma. Br J Ophthalmol 1940; 24: 288-297.

2 Bietti G. Surgical intervention on the ciliary body; new trends for the relief of glaucoma. JAMA 1950; 142: 889-897.

3 Hampton C, Shields MB, Miller KN, Blasini M. Evaluation of a protocol for trans-scleral neodymium: YAG cyclophotocoagulation in one hundred patients. Ophthalmology 1990; 97: 910-917.

4 Peyman GA, Naguib KS, Gaasterland D. Trans-scleral application of semiconductor diode laser. Lasers Surg Med 1990; 10: 569-575.

5 Assia EI, Hennis HL, Stewart WC, Legler UF, Carlson AN, Apple DJ. A comparison of neodymium:yttrium aluminium garnet and diode laser trans-scleral cyclophotocoagulation and cyclocryotherapy. Invest Ophthalmol Vis Sci 1991; 32: 2774-2778.

6 Hennis HL, Stewart WC. Semiconductor diode laser trans-scleral cyclophotocoagulation in patients with glaucoma. Am J Ophthalmol 1992; 113: 81-85.

7 Schuman JS, Noecker RJ, Puliafito CA, Jacobson JJ, Shepps GJ, Wang N. Energy levels and probe placement in contact transscleral semiconductor diode laser cyclophotocoagulation in human cadaver eyes. Arch Ophthalmol 1991; 109: 1534-1538.

8 Hennis HL, Assia E, Stewart WC, Legler UF, Apple DJ. Transscleral cyclophotocoagulation using a semiconductor diode laser in cadaver eyes. Ophthalmic Surg 1991; 22: 274-278.

9 Murphy CC, Burnett CA, Spry PG, Broadway DC, Diamond JP. A two centre study of the dose-response relation for transscleral diode laser cyclophotocoagulation in refractory glaucoma. Br J Ophthalmol 2003; 87: 1252-1257.

10 Spencer AF, Vernon SA. Cyclodiode results of a standard protocol. Br J Ophthalmol 1999; 83: 311-316.

11 Bloom PA, Tsai JC, Sharma K, Miller MH, Rice NS, Hitchings RA et al. Cyclodiode trans-scleral diode laser cyclophotocoagulation in the treatment of advanced refractory glaucoma. Ophthalmology 1997; 104: 1508-1520.

12 Allingham RR, de Kater AW, Bellows AR, Hsu J. Probe placement and power levels in contract transscleral neodymium:YAG cyclophotocoagulation. Arch Ophthalmol 1990; 108: 738-742.

13 Fiore PM, Latina MA. A technique for precise placement of laser applications in transscleral Nd:YAG cyclophotocoagulation. Am J Ophthalmol 1989; 107: 292-293.

14 Agrawal P, Martin KR. Ciliary body position variability in glaucoma patients assessed by scleral transillumination. Eye 2008; 22: 1499-1503.

15 Uram M. Endoscopic flourescein angiography of the ciliary body in glaucoma management. Ophthalmic Surg Lasers 1996; 27: 174-178.

16 Rebolleda G, Munoz FJ, Murube J. Audible pops during cyclodiode procedures. J Glaucoma 1999; 8: 177-183.

17 Kaushik A, Pandav SS, Jain R, Bansal S, Gupta A. Lower energy levels adequate for effective transscleral diode laser cyclophotocoagulation in Asian eyes with refractory glaucoma. Eye 2008; 22: 398-405.

18 Vernon SA, Koppens JM, Menon GJ, Negi AK. Diode laser cycloablation in adult glaucoma: long-term results of a standard protocol and review of current literature. Clin Experiment Ophthalmol 2006; 34: 411-420.

19 Cantor LB, Nichols DA, Katx LJ, Moster MR, Poryzees E, Shields JA et al. Neodymium-YAG transscleral cyclophotocoagulation. The role of pigmentation. Invest Ophthalmol Vis Sci 1989; 30: 1834-1837.

20 Leon C, Leon J. The ciliary body. In: Leon C, Leon J (eds). Endoscopic Chirurgicale Oculaire. Medsi/Mcgraw-Hill: Paris, France, 1990, pp 42-47.

21 Gaasterland DE, Pollack IP. Initial experience with a new method of laser transscleral cyclophotocoagulation for ciliary ablation in severe glaucoma. Trans Am Ophthalmol Soc 1992; 90: 225-243.

22 Kosoko O, Gaasterland DE, Pollack IP, Enger CL. The Diode Laser Ciliary Ablation Study Group. Long-term outcome of initial ciliary ablation with contact diode laser transscleral cyclophotocoagulation for severe glaucoma. Ophthalmology 1996; 103: 1294-1302.

23 Five-year follow-up of the Fluorouracil Filtering Surgery Study. The Fluorouracil Filtering Surgery Study Group. Am J Ophthalmol 1996; 121: 349-366.

24 Siriwardena D, Kotecha A, Minassian D, Dart JK, Khaw PT. Anterior chamber flare after trabeculectomy and after phacoemulsification. Br J Ophthalmol 2000; 84: 1056-1057.

25 Crowston JG, Wang XY, Khaw PT, Zoellner H, Healey PR. Human serum reduces mitomycin-C cytotoxicity in human tenon's fibroblasts. Invest Ophthalmol Vis Sci 2006; 47: 946-952. 